Ann. Zootech., I963, 12 (3), I93-202.

\title{
EFFET DE LA CONDUITE DU SÉCHAGE A LA FLAMME SUR LA VALEUR BIOLOGIQUE DE FARINES DE HARENGS CHEZ LE PORC
}

\author{
J. DELORT-LAVAL et S. Z. ZELTER \\ avec la collaboration technique de P. Durbant et Michèle FiszLewicz \\ Laboratoire de Recherches sur la Conservation et l'Efficacité des Aliments, \\ I6, rue Claude-Bernard, Paris-5
}

\section{SOMMAIRE}

Le séchage à la flamme peut modifier l'efficacité azotée des farines de harengs, en raison de l'intensité du traitement thermique appliqué.

Pour tester l'influence de ce facteur, trois farines (désignation : 60, 90, I30), préparées dans des conditions aussi homogènes que possible par ailleurs, ont été séchées à des températures différentes et distribuées comme seule source azotée à des porcs.

Les coefficients d'utilisation digestive apparente $(86,5 ; 85,3 ; 85,3)$ et réelle $(94,4 ; 94,7 ; 94,5)$ et les valeurs biologiques $(60,0 ; 6 \mathrm{r}, 3 ; 6 \mathrm{r}, 6)$ de ces trois produits, mesurés selon le principe de la rotation des régimes sur six animaux par la technique de THovas-MrTchell, ne diffèrent pas significativement. L'épreuve de la solubilité à la pepsine chlorhydrique et le calcul des index de qualité d'Oser et de Mrrchell, à partir de la composition en acides aminés, ne permettent pas non plus de distinguer les trois farines.

\section{IN'TRODUC'TION}

L'influence considérable du traitement thermique sur l'efficacité azotée des farines de poisson a été mise en évidenze dans des travaux déjà anciens, passés en revue par CREAC'H (I950), GRAU et WiLLIAMS (I955), OUSTERHOUT et SNYDER(I962). Les premières recherches ont montrél'action nocive du séchage à la flamme (WIL,Gus et $a l$., I935). Mais l'infériorité de la qualité des produits ainsi préparés peut avoir pour cause, en dehors de la dénaturation des protéines par le traitement thermique, une destruction plus ou moins intense des vitamines du groupe B (Clandinin, I949; BiELy et al., I952; KıUNGSOYR et al., I953). L'amélioration dar ovuutions expérimentales d'éva- 
luation de l'efficacité azotée a permis de montrer que, grâce aux progrès de la technologie, il est devenu possible d'obtenir, par ce procédé, des produits comparables aux farines séchées sous vide ou à la vapeur (TARR et al., I954; CARPENTER et al., I954).

Mais la qualité variable des farines de poisson ne dépend pas du seul mode de séchage. De nombreux autres tacteurs peuvent modifier de façon déterminante la valeur azotée du produit final : origine, composition (RAND, I960) et état de fraîcheur de la matière première, durée et température de la cuisson précédant le séchage, échauffement spontané des farines à la sortie du séchoir (BOGE, I960), conditions de stockage (LEA et al., I960).

Dans le but d'étudier l'effet de la température de séchage sur l'efficacité azotée des farines de harengs, 3 échantillons préparés dans des conditions aussi homogènes que possible pour éviter l'interférence des facteurs de variations meationn s ci-dessus, ont été séchées à la flamme, à des températures différentes. Leur valeur biologique mesurée sur le porc a été comparée aux résultats de quelques épreuves biochimiques mises en œuvre simultanément.

\section{MATÉRIEI, E'T MÉTHODES}

\section{A. - Caractéristiques des farines de harengs}

\section{Technologie}

Les trois farines ont été préparées, à partir d'un même lot de pêche de harengs d'hiver, par voie humide et sans réincorporation de la fraction soluble séparée au cours du pressage, dans l'usine-pilote de l'Institut de Recherches sur les huiles et farines de poisson de T'jaereviken (Norvège).

TABLEAU I

Conditions de séchage des farines de harengs

\begin{tabular}{|c|c|c|c|c|}
\hline \multirow{2}{*}{ Fichantillon } & \multicolumn{2}{|c|}{ Température des gaz ( ${ }^{\circ}()$} & \multirow{2}{*}{$\begin{array}{l}\text { Mrmission } \\
\text { d'air secondaire }\end{array}$} & \multirow{2}{*}{$\begin{array}{l}\text { Ilumidité } \\
\text { des farines }\end{array}$} \\
\hline & Admission & Sortic & & \\
\hline $60 \ldots$ & $490-520$ & 60 & Cormale & 9,1 \\
\hline $90 \ldots \ldots \ldots \ldots \ldots \ldots$ & 750 & 90 & Rérluite & 9,1 \\
\hline $130 \ldots \ldots \ldots$ & 600 & 130 & Accrue & 10,2 \\
\hline
\end{tabular}

Les trois produits ont été séchés à la flamme diuns les conditions de températures et de durées indiquées au tableau I ; ils sont désignés par un nombre correspondant à leur température de sortie du four. Il n'y a pas de correspondance directe entre cette donnée el la température à l'entrée du séchoir. Ia quantité d'air secondaire admise, facteur qui détermine le temps de transit et donc la durée du chauffage, n'est malheureusement pas connue de façon précise. ressat.

A leur arrivéc en France, les farines ont été conservées $\mathrm{I} 2$ mois à $\rightarrow$ I $5^{\circ} \mathrm{C}$ avant le début de 


\section{$2^{\circ}$ Composition}

La composition des farines de harengs est indiquée dans le tableau 2.

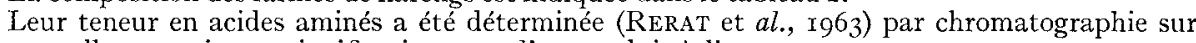
colonne; elle ne varie pas significativement d'un produit à l'autre.

TABLEAU 2

Composition des aliments

\begin{tabular}{|c|c|c|c|c|c|c|}
\hline Produit & $\begin{array}{c}\text { Humidité } \\
(\%)\end{array}$ & $\begin{array}{c}\text { Matières } \\
\text { minérales } \\
(\%)\end{array}$ & $\begin{array}{c}\text { Matières } \\
\text { azotées } \\
(\mathrm{N} \times 6,25) \\
(\%)\end{array}$ & $\begin{array}{c}\text { Matières } \\
\text { grasses } \\
\text { (extraction } \\
\text { à l'éther) } \\
(\%)\end{array}$ & $\begin{array}{c}\text { Acidité } \\
\left(\mathrm{g} \mathrm{SO}_{4} \mathrm{H}_{2}\right) \\
(\%)\end{array}$ & $\begin{array}{c}\text { Énergie } \\
\text { brute } \\
\mathrm{cal} / \mathrm{g} \text { M.S. }\end{array}$ \\
\hline Farine 60. & 9,1 & 10,1 & 72,7 & 6,5 & 0,57 & 5370 \\
\hline Farine 90. & 9,1 & 9,7 & 72,8 & 6,9 & 0,58 & 5450 \\
\hline Farine 130. & 10,2 & 9,6 & 70,9 & 7,2 & 0,61 & 5350 \\
\hline Aliment ternaire. & 0 & 6,5 & 0,3 & 3,2 & - & 3820 \\
\hline
\end{tabular}

B. - Prótocole EXPÉRIMENTAL,

\section{$\mathbf{I}^{\circ}$ Animaux}

Six porcs mâles castrés de race Large White (E F G H I J) proviennent de deux portées différentes mais ont le même père. Au début de l'expérience, ils sont respectivement âgés de $92(\mathrm{E} \mathrm{F} \mathrm{G})$ et 88 ( $\mathrm{H} \mathrm{I} \mathrm{J)} \mathrm{jours} \mathrm{et} \mathrm{leurs} \mathrm{poids} \mathrm{s'échelonnent} \mathrm{de} 24,7$ à $33,7 \mathrm{~kg}$.

\section{$2^{\mathrm{O}}$ Régime alimentaire}

Le régime de base (DELORT-LAVAL et al., i 963) est un aliment énergétique pratiquement dépourvu d'azote $(0,47$ à $0,54 \mathrm{~g}$ par kg de matière sèche) mais équilibré par ailleurs, notamment en vitamines du groupe $B$.

Il est complété selon les cas par l'une des trois farines de harengs, de manière à atteindre un taux d'environ Ioo g de matières azotées digestibles par unité fourragère (m. a. d./u. f.), soit I2,8 p. I00 de matières azotées totales par kg de matière sèche.

La ration journalière calculée sur la base de 4 p. ıoo du poids vif de l'animal est distribuée en 2 fois. Les refus, le plus souvent insignifiants, sont pesés après chaque repas.

\section{$3^{\circ}$ Collecte des excreta}

Les animaux sont placés en cages à métabolisme individuelles. Ils sont en outre munis d'appareils destinés à la collecte de l'urine (Zelter et Charlet-Lery, 196I), qui évitent les contaminations et les pertes par évaporation. L'urine est recueillie dans des bocaux sous acide sulfurique. Des sacs en polyéthylène, changés deux fois par jour, sont fixés à la partie postérieure de l'animal par un système de harnais ; ils facilitent grandement la récolte des fèces.

\section{$4^{\circ}$ Dispositif expérimental}

La digestibilité apparente de l'azote est mesurée au cours d'une première période $\left(\mathrm{R}_{\mathrm{I}}\right)$; cette donnée sert à ajuster, dans la suite de l'essai, le taux de m. a. d./u. f. du régime distribué. L'expérience se poursuit par i 6 jours de jeûne protidique, dont les 8 derniers servent à l'estimation des dépenses 
endogènes. Après une phase de réalimentation de 20 jours, temps suffisant pour le rétablissement d'un métabolisme normal de l'azote (DELORT-LAVAL et al., I963), les trois farines sont distribuées, selon le principe de la rotation des régimes, aux 6 porcs durant 3 périodes successives de bilan $R_{I I}$, $\mathrm{R}_{\mathrm{III}} \mathrm{R}_{\mathrm{IV}}$, (tableau 3) séparées par au moins 8 jours d'adaptation.

\section{TABLEAU 3}

Schéma expérimental el répartition des farines de harengs durant les périodes de bilan

\begin{tabular}{|c|c|c|c|c|c|c|}
\hline $\begin{array}{cc} & \text { Animal } \rightarrow \\
\text { Période } & \\
\downarrow & \end{array}$ & $\mathrm{E}$ & $\mathrm{F}$ & G & $\mathrm{H}$ & I & $\mathrm{J}$ \\
\hline $\mathrm{R}_{\mathrm{I}} \ldots \ldots \ldots \ldots \ldots$ & 60 & 130 & 90 & 130 & 60 & 90 \\
\hline $\mathrm{D}$ & \multicolumn{6}{|c|}{ Régime protéiprive } \\
\hline $\mathrm{R}_{\mathrm{II}} \ldots \ldots \ldots \ldots \ldots$ & 130 & 90 & 60 & 60 & 90 & 130 \\
\hline$\overline{R_{I I I}} \ldots \ldots \ldots \ldots \ldots$ & 90 & 60 & 130 & 90 & 130 & 60 \\
\hline $\mathrm{R}_{I V} \ldots \ldots$ & 60 & 130 & 90 & 130 & 60 & 90 \\
\hline
\end{tabular}

La teneur en azote des échantillons d'aliments, de fèces frais et d'urine est dosée par la méthode de KJELDAHL (1). La valeur calorique de l'aliment et des fèces est mesurée par bombe calorimétrique et sert à déterminer la digestibilité de l'énergie des rations.

Le calcul de la valeur biologique, à partir des données de la période protéiprive, s'effectue sur la base des résultats de travaux antérieurs (ZELTER et CHARLET-LERY, I96I). L'azote métabolique fécal est rapporté à Ia matière sèche fécale et l'azote endogène urinaire à la taille métabolique $\left(\mathrm{P}^{0,70}\right.$, après correction due à la différence d'âge entre les jours moyens des périodes de bilan en réplétion et en déplétion azotée.

\section{$5^{0}$ Tests biochimiques}

Les 3 échantillons sont soumis à des tests de solubilité en milieu pepsine-HCl. L'épreuve s'effectue à $\mathrm{pH} \mathrm{r}, 7$ avec agitation, sur $\mathrm{I} g$ de produit, en présence de $50 \mathrm{mg}$ de pepsine (B. L. B., pouvoir $200)$, ̀̀ deux températures $\left(40^{\circ} \mathrm{C}\right.$ et $\left.45^{\circ} \mathrm{C}\right)$ et deux durées différentes $(6 \mathrm{~h}$ et $20 \mathrm{~h})$.

L'index de qualité des protéines animales est déterminé selon la méthode proposée par ALMQuisT et STACKSTA (1935). En outre, à partir de la composition en acides aminés (RERAT et al., 1963), ont été calculés la "classe chimique "(Mrtchell et BLock, 1946) et l'index d'acides aminés essentiels (OSER, I959).

\section{RÉSULTATS}

Les donnóes des cinq périodes de bilans sont groupées dans le tableau 4. Durant les trois dernières périodes, les gains de poids journaliers, compris entre 560 et $890 \mathrm{~g}$, sont normatix. Les coefficients d'utilisation digestive apparente (C. U. D. A.) et réelle

(1) Catalyseur : oxyde rouge de mercure ; micro-distillation dans l'appareil de Parnas-Wagner ; distillat recueilli dans une solution d'acide borique I p. 100 et titré par $\mathrm{SO}_{4} \mathrm{H}_{2}, 0,02 \mathrm{~N}$ en présence de l'indicateur de CONWAY. 
(C. U. D. R.) de l'azote, les valeurs biologiques (V. B.) et l'utilisation protidique nette (V. B. $\times$ C. U. D. R./IOo) des 3 farines ne diffèrent pas significativement (tablea11 5).

\section{TABLEAU 4}

Résultats moyens journaliers par période et par animal

\begin{tabular}{|c|c|c|c|c|c|c|c|c|c|c|}
\hline Période & $\begin{array}{c}\text { Animal } \\
\text { Farine }\end{array}$ & $\begin{array}{l}\text { Poids } \\
\text { (kg) }\end{array}$ & $\begin{array}{c}\text { Crois- } \\
\text { sance } \\
\text { (g/jour) }\end{array}$ & $\begin{array}{c}\text { M.S.i. } \\
\left({ }^{1}\right)\end{array}$ & $\begin{array}{l}\text { M.S.i. } \\
\text { / Po,zo }\end{array}$ & $\begin{array}{c}\text { C.U.D. (1) } \\
\text { énergie } \\
(\%)\end{array}$ & $\begin{array}{c}\mathrm{N} \\
\text { ingéré } \\
(g)\end{array}$ & $\begin{array}{c}\mathrm{N} \\
\text { urinaire } \\
\text { (g) }\end{array}$ & $\begin{array}{c}X \\
\text { fecal } \\
(\mathrm{g})\end{array}$ & $\begin{array}{l}\text { N Bilan } \\
\text { apparent } \\
\text { (g) }\end{array}$ \\
\hline $\mathrm{R}_{\mathbf{I}}$ & $\begin{array}{l}\text { E. } 60 \\
\text { F. } 130 \\
\text { G. } 90 \\
\text { H. } 130 \\
\text { I. } 60 \\
\text { J. } 90\end{array}$ & $\begin{array}{l}38,0 \\
37,3 \\
36,4 \\
31,0 \\
24,8 \\
27,4\end{array}$ & $\begin{array}{l}0,527 \\
0,423 \\
0,459 \\
0,238 \\
0,243 \\
0,269\end{array}$ & $\begin{array}{r}1310 \\
1290 \\
1251 \\
1085 \\
807 \\
966\end{array}$ & $\begin{array}{r}105,0 \\
102,5 \\
100,2 \\
98,0 \\
85,3 \\
95,2\end{array}$ & & $\begin{array}{l}24,92 \\
24,33 \\
23,51 \\
21,32 \\
17,27 \\
18,11\end{array}$ & & $\begin{array}{l}3,01 \\
3,38 \\
3,25 \\
3,16 \\
2,54 \\
2,80\end{array}$ & \\
\hline $\mathrm{D}$ & $\begin{array}{l}\mathrm{E} \\
\mathbf{F} \\
\mathrm{G} \\
\mathrm{H} \\
\mathrm{I} \\
\mathrm{J}\end{array}$ & $\begin{array}{l}41,4 \\
40,0 \\
39,7 \\
32,4 \\
27,2 \\
29,5\end{array}$ & $\begin{array}{l}0,070 \\
0,000 \\
0,040 \\
0,040 \\
0,050 \\
0,080\end{array}$ & $\begin{array}{l}1480 \\
1415 \\
1434 \\
1164 \\
1020 \\
1179\end{array}$ & $\begin{array}{l}109,1 \\
107,0 \\
109,0 \\
102,0 \\
100,5 \\
110,0\end{array}$ & $\begin{array}{l}87,7 \\
87,1 \\
86,0 \\
85,2 \\
81,6 \\
87,1\end{array}$ & $\begin{array}{l}0,77 \\
0,74 \\
0,75 \\
0,61 \\
0,54 \\
0,61\end{array}$ & $\begin{array}{l}2,50 \\
2,28 \\
2,10 \\
1,91 \\
1,82 \\
1,81\end{array}$ & $\begin{array}{l}2,60 \\
2,52 \\
0,75 \\
2,30 \\
2,06 \\
2,14\end{array}$ & $\begin{array}{r}4,33 \\
-\quad 4,06 \\
-\quad 4,18 \\
-\quad 3,60 \\
-\quad 3,37 \\
-\quad 3,34\end{array}$ \\
\hline $\mathrm{R}_{\text {II }}$ & $\begin{array}{l}\text { E. } 130 \\
\text { F. } 90 \\
\text { G. } 60 \\
\text { H. } 60 \\
\text { I. } 90 \\
\text { J. } 130\end{array}$ & $\begin{array}{l}61,9 \\
59,7 \\
57,0 \\
47,2 \\
39,9 \\
44,0\end{array}$ & $\begin{array}{l}0,817 \\
0,880 \\
0,812 \\
0,599 \\
0,563 \\
0,637\end{array}$ & $\begin{array}{l}2092 \\
1989 \\
1940 \\
1620 \\
1335 \\
1470\end{array}$ & $\begin{array}{l}116,5 \\
113,5 \\
114,5 \\
109,0 \\
101,1 \\
104,0\end{array}$ & $\begin{array}{l}86,8 \\
87,1 \\
87,9 \\
85,5 \\
85,3 \\
86,6\end{array}$ & $\begin{array}{l}45,99 \\
43,66 \\
42,67 \\
35,30 \\
29,69 \\
32,29\end{array}$ & $\begin{array}{r}17,20 \\
15,58 \\
16,06 \\
12,15 \\
10,42 \\
9,27\end{array}$ & $\begin{array}{l}5,97 \\
5,24 \\
4,87 \\
4,97 \\
4,51 \\
4,85\end{array}$ & $\begin{array}{l}22,8: \\
22,8 ' t \\
21,5 \prime \\
18,18 \\
14,76 \\
18,17\end{array}$ \\
\hline$R_{\text {III }}$ & $\begin{array}{l}\text { E. } 90 \\
\text { F. } 60 \\
\text { C. } 130 \\
\text { H. } 90 \\
\text { I. } 130 \\
\text { J. } 60\end{array}$ & $\begin{array}{l}76,3 \\
74,4 \\
70,8 \\
61,9 \\
51,3 \\
55,6\end{array}$ & $\begin{array}{l}0,821 \\
0,888 \\
0,808 \\
0,787 \\
0,685 \\
0,631\end{array}$ & $\begin{array}{l}2439 \\
2345 \\
2187 \\
2087 \\
1769 \\
1810\end{array}$ & $\begin{array}{l}117,3 \\
111,8 \\
110,9 \\
115,8 \\
112,3 \\
108,6\end{array}$ & $\begin{array}{l}87,0 \\
88,2 \\
88,6 \\
81,4 \\
83,9 \\
85,0\end{array}$ & $\begin{array}{l}50,92 \\
48,15 \\
45,02 \\
43,60 \\
36,07 \\
37,08\end{array}$ & $\begin{array}{l}25,68 \\
21,16 \\
18,72 \\
11,40 \\
16,8 \\
17,56\end{array}$ & $\begin{array}{l}7,59 \\
6,04 \\
5,72 \\
8,26 \\
6,18 \\
6,0\end{array}$ & $\begin{array}{l}17,65 \\
20,65 \\
20,58 \\
20,99_{k}^{\prime} \\
12,75 \\
13,52\end{array}$ \\
\hline $\mathrm{R}_{\text {IV }}$ & $\begin{array}{l}\text { E. } 60 \\
\text { F. } 130 \\
\text { G. } 90 \\
\text { II. } 130 \\
\text { I. } 60 \\
\text { J. } 90\end{array}$ & $\begin{array}{l}88,7 \\
85,7 \\
80,7 \\
72,6 \\
60,7 \\
65,9\end{array}$ & $\begin{array}{l}0,728 \\
0,659 \\
0,68 / \\
0,705 \\
0,579 \\
0,651\end{array}$ & $\begin{array}{l}257 ! \\
2 ! 34 \\
2379 \\
2333 \\
2025 \\
21_{4}^{\prime} 7\end{array}$ & $\begin{array}{l}111,4 \\
117,9 \\
110,0 \\
116,1 \\
111,3 \\
111,4\end{array}$ & $\begin{array}{l}89,3 \\
89,1 \\
87,1 \\
86,9 \\
86,8 \\
85,5\end{array}$ & $\begin{array}{l}52,03 \\
50,24 \\
50,12 \\
39,54 \\
40,90 \\
15,34\end{array}$ & $\begin{array}{l}30,40 \\
26,51 \\
26,81 \\
20,92 \\
19,40 \\
20,78\end{array}$ & $\begin{array}{l}6,98 \\
6,57 \\
6,77 \\
7,58 \\
5,50 \\
6,2 \text { ' }\end{array}$ & $\begin{array}{l}14,65 \\
17,16 \\
16,54 \\
11,01 \\
16,00 \\
18,32\end{array}$ \\
\hline
\end{tabular}

(1) M. S. i. : matière sèche ingérée ; C. U. D. : Coefficient d'utilisation digestive.

Les résultats des tests biochimiques (tableau 6), solubilité pepsique, classe chimique, index d'acides aminés essentiels, ne permettent pas non plus de distinguer les 3 produits ; seul l'index de qualité des protéines varie d'un échantillon à l'autre. 
TABLEAU 5

Efficacité azotée des farines de harengs
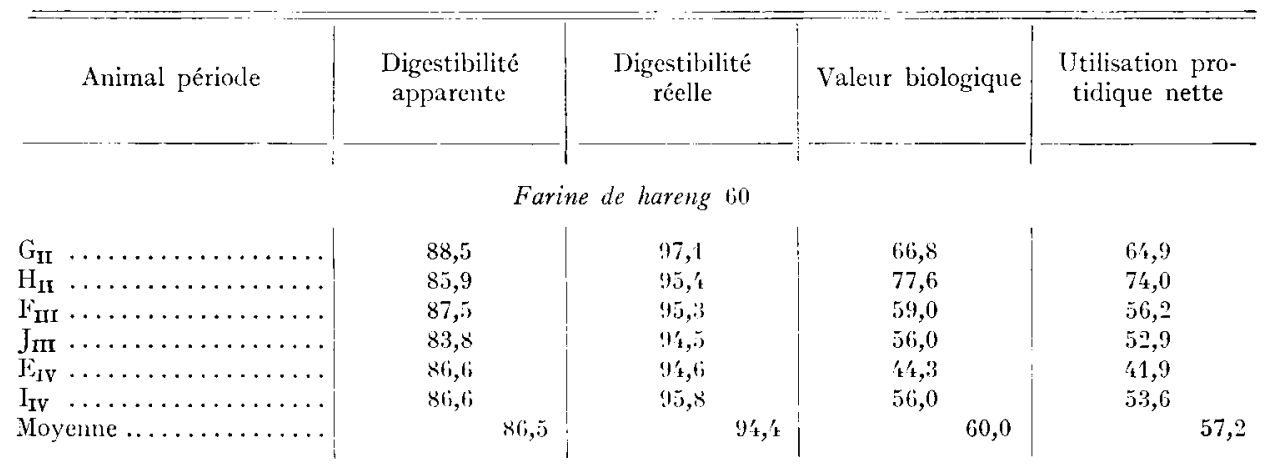

Farine de hareng 60

88,5
85,9
87,5
83,8
86,6
86,6

97,1
95,4
95,3
91,5
94,6
95,8

$\begin{array}{ll}66,8 & \\ 77,6 & \\ 59,0 & \\ 56,0 & \\ 14,3 & \\ 56,0 & \\ & 60,0\end{array}$

6,9

74,0

56,2

5 으, 9

41,9

53,6

Farine de hareng 90

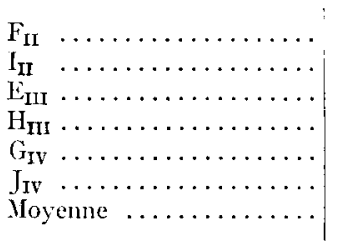

88,0

8,8

85,1

81,0

86,5

86,2

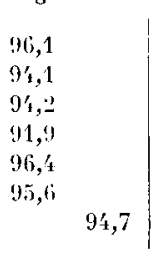

$\begin{array}{ll}69,0 & \\ 69,9 & \\ 52,7 & \\ 69,7 & \\ 49,6 & \\ 57,0 & \\ & 61,3\end{array}$

66,3

65,8

19,6

64,0

47,8

54,5

58,0

Farine de haveng 130

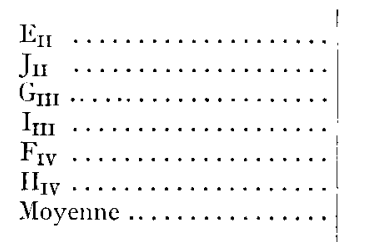

87,0
85,0
87,3
82,0
86,9
85,9

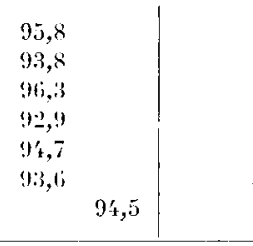

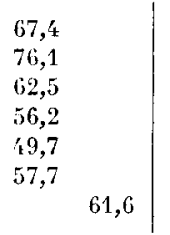

64,6

71,4

60,2

52,2

47,1

54,0

\section{TABLEAU 6}

Tests biochimiques d'évaluation de la qualité des protides de farines de harengs

\begin{tabular}{|c|c|c|c|c|c|c|c|}
\hline \multirow{3}{*}{ Farines } & \multicolumn{4}{|c|}{ Solubilité pepsine $\mathrm{ICl}$ à pHI 1,7} & \multirow{3}{*}{$\begin{array}{l}\text { Index } \\
\text { d'acides } \\
\text { aminés } \\
\text { essentiels } \\
\text { (OsNR) }\end{array}$} & \multirow{3}{*}{$\begin{array}{c}\text { Classe } \\
\text { chimique } \\
\text { (MrTcillLL } \\
\text { et Brock) }\end{array}$} & \multirow{3}{*}{$\begin{array}{l}\text { Index de } \\
\text { qualité } \\
\text { (ALMQuist) }\end{array}$} \\
\hline & \multicolumn{2}{|c|}{$6 \mathrm{~h}$} & \multicolumn{2}{|c|}{$20 \mathrm{~h}$} & & & \\
\hline & 1000 & $45^{\circ} \mathrm{C}$ & ${ }^{\prime} 0^{\circ} \mathrm{C}$ & $15 \% \mathrm{C}$ & & & \\
\hline 60) & 86,8 & 88,1 & 90,2 & 89,7 & 85,6 & 68,1 & 67 \\
\hline 90 & 85,1 & 87,3 & 89,5 & 90.1 & 81,7 & 68,4 & 75 \\
\hline 130 & 81,3 & 88,1 & 89,2 & 901,8 & 83,6 & $68, \mathbf{1}$ & 82 \\
\hline
\end{tabular}




\section{DISCUSSION}

Les coefficients d'utilisation digestive de l'énergie des rations et de l'azote des farines de harengs ne diffèrent pas sensiblement d'une période à l'autre. La comparaison des valeurs moyennes montre que, dans les limites de notre étude, l'influence du traitement thermique sur la digestibilité des farines est uniforme.

Les valeurs biologiques sont plus délicates à interpréter. Ė̀n effet, la distribution d'un aliment en quantité croissante et au taux constant de roo g. m. a. d/u. f. conduit à une augmentation du rapport $\mathrm{N}$ ingéré dépense azotée endogène minimum au cours de l'essai. La décroissance de la valeur biologique qui s'ensuit (Columbus, I950) est accentuée par la progression simultanée du poids de l'animal (HoHLs, I955). La figure I met nettement en évidence cette décroissance de la valeur biologique entre 30 et $90 \mathrm{~kg}$.

L'analyse de covariance destinée à réduire l'effet du poids sur la valeur bioiogique confirme que le dispositif expérimental adopté au cours de l'essai corrige efficacement les variations individuelles dues surtout à ce facteur. Il est donc possible de comparer valablement les moyennes des valeurs biologiques : elles sont pratiquement similaires : respectivement 60,0,6I,3,6r,6 pour les farines 60 , 90 et I30.

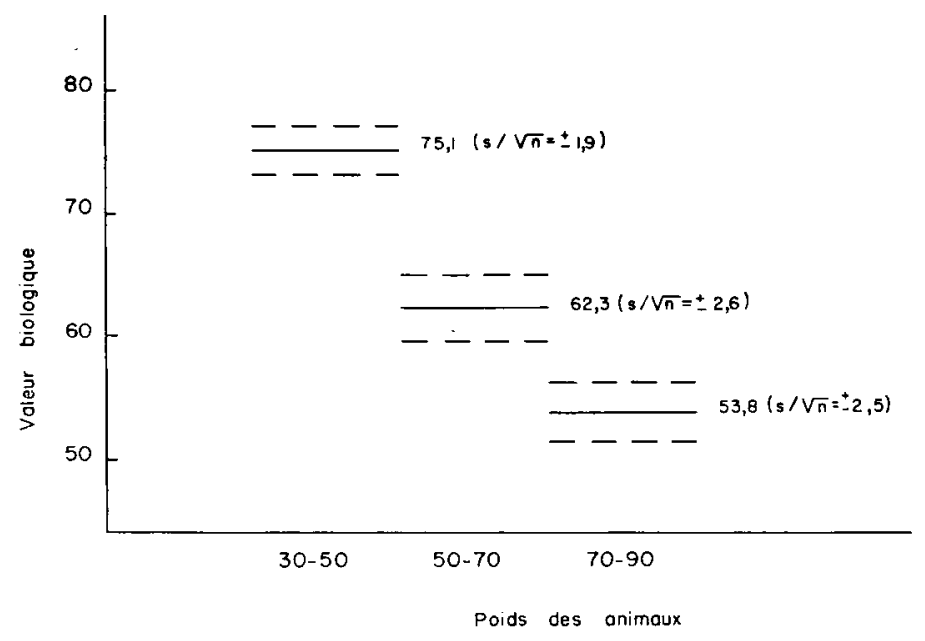

FIG. I. - Lvolution de la valeur biologique en fonction du poids des animaux

Les coefficients d'utilisation digestive de l'azote et les valeurs biologiques des farines de harengs cités dans la littérature sont groupés danミ le tableau 7. On contate que, chez le porc, NEHRING et LAUBE (I96r) obtiennent un résultat très voisin des nôtres, tandis que la valeur biologique du même produit mesurée sur le rat est nettement inférieure. Cette donnée paraît toutefois anormalement basse en comparaison de celles obtenues par ailleurs sur le rat (tableau 7 ). 
La valeur moyenne enregistrée sur nos porcs entre 30 et $50 \mathrm{~kg}$ (fig. I) se rapproche sensiblement de ces dernières.

De l'examen des résultats des tests biochimiques d'évaluation de la qualité des protéines (tableau 6), i1 ressort que :

- la solubilité pepsique fournit des valeurs comparables entre elles et toujours inférieures aux digestibilités réelles mesurées sur l'animal;

- la très importante variation de l'index de qualité d'Almourst et STAcksta (I935) n'est pas confirmée in vivo, ce qui diminue considérablement l'intérêt de ce critère, de détermination analytique par ailleurs délicate.

TABLEAU 7

Données bibliographiques sur la valcur biologique de farines de harengs

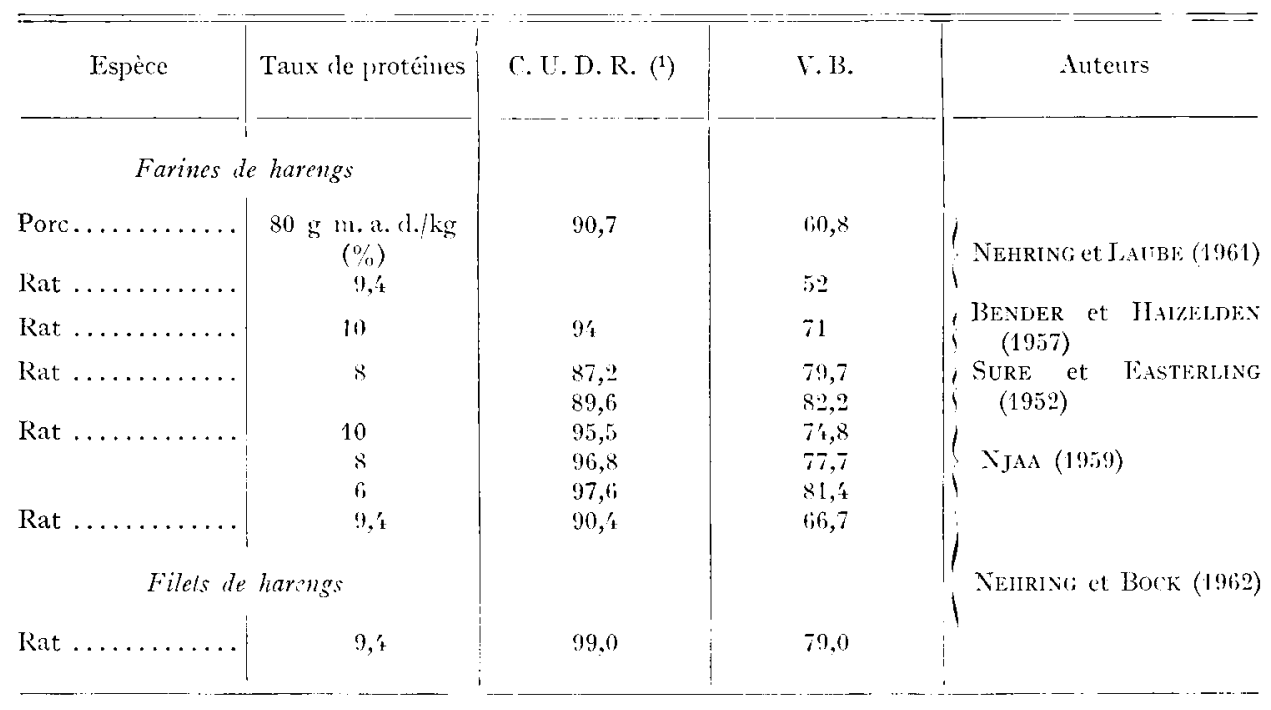

(1) C. U. D. R. : coefficient d'utilisation digestive réelle de l'azote.

La prévision de la valeur biologique à partir de l'index d'OSER (I959) procure des valeurs plus élevées $(82,77$ et 79 respectivement pour les farines 60 , 90 et I30) que nos données in vivo, même les plus fortes. Le calcul effectué à partir de la classe chimique par la formule de MITCHELL et BI.OCK (I946) fournit également des résultats plus élevés, identiques (82) pour les trois farines.

Dans les limites des conditions de séchage à la flamme mises en ouvre pour la fabrication de nos trois farines de harengs, il n'a donc été possible, ni par la mesure de la valeur biologique sur le porc, ni au moyen de tests biochimiques, de mettre en évidence une différence entre les trois produits. 


\section{REMERCIEMENTS}

Nous remercions le Sildolje-og Sildemelindustriens Forskningsinstitutt pour la préparation des échantillons de farines de harengs et l'organisation norvégiemne sildenelutvalget pour le concours financier qu'elle a apporté ì la réalisation de cette étude.

\section{SUMMARY}

\section{THE EFFECT OF THE FLANE DRYING PROCESS ON THE BIOLOGICAL VALUE OF HERRING MEAL IN THE PIG}

The intensity of the heat treatment employed in flame drying can modify the nitrogen efficiency of herring meal.

'To test the influence of this factor, three meals (designated 60, 90, I30) prepared under as similar conditions as possible (table $\mathrm{I}$ ) were dried out at different temperatures and given to pigs as the sole source of nitrogen.

The coefficients of apparent digestibility $(86.5 ; 85.3 ; 85.3)$, true digestibility $(94.4 ; 94.7$; $94.5)$ and the biological values $(60.0 ; 6 \mathrm{r} .3 ; 6 \mathrm{I} .6)$ of the three products, measured according to the latin square system by the Thomas-MITcheic technique on six animals, do not differ significantly from each other (table 5). The determination of solubility in $\mathrm{IICl}$ pepsin and the calculation of the quality indexes of OSER and MícineLL do not permit any distinction to be made between the three meals (table 6).

\section{RÉFÉRENCES BIBLIOGRAPHIQUES}

Almouist H. J., Stacksta D., I935. Supplementary values of animal protein in chick rations. J. Nutr., 10, I93-2II.

Bender A. T., HatzedueN Sheila, I957. Biological value of the proteins of a variety of fish meals. Brit. J. Nutr., 11, 42-43.

Biely J., March B. E., Tarr H. L. A., I952. 'The nutritive value of fish meal and condensed fish solubles. IVe Pros. Rep. Pacific Coast Sta., 90, 10-13.

Boge G., r 960 . Amino-acid composition of herring (Clupea harengus) and herring meal. Destruction of amino-acids during processing. J.Sci. Food Agric., 11, 362-365.

Carpenter G., Elimangr M., Surimpton D. H., i 954 . 'The nutritive value of six white fish meals of known origin. Proc. Nutr. Soc., 13, 20-21.

Clantinis D. R., i949. The effects of methods of processing on the nutritive value of herring meals. Poultry Sci., 28, I $28-133$.

Columbus A., I950. Die Variabilität der biologischen Eiweisswertigheit unter verschiedenen Bedingungen nach Stoffwechselversuchen an wachsenden Schweinen. Arch. Tiprernäh., 1, 38-55;84-ı 28.

Creac'II P. V., 1950. Congrès International d'étules sur le rôle du poisson dans l'alimentation, 249-204, Institut Océanographique, Paris.

Deiort-Laval. J., Chardet-Lery Geneviève, Dogan K., ig63. Efficacité de quelques protides alimentaires chez le porc. III. Évolution de l'excrétion azotée urinaire durant la phase de réalimentation après un jeûne protéique. Ann. Biol. anim. Bioch. Biophys., 3, 255-262

GraU C. R., Wildams H. A., 1955. Fish meals as amino-acid sources in chick rations. Poultry Sci., 34, $810-817$.

Honlls II. W., 1955. Die Berechnung des maximalen täglicher Eiweissansatz-vermögens von Schweinen aus in der Litteratur veröffentlichten Daten und dessen Berleutung bei der Durchführung von Fütterungsversuchen. Z. Tievernähr., 10, 44-55.

Klungsoyr M.,Boge r., Sparre T', i953. Séchage de farines. (Norvegien) Meldingerfra S. S. F., Damsgard, Bergen, 2, 22-30.

Lea C. I., PARr L. S., Carpenter K. J., 1960. Chemical and nutritional changes in stored herring meal. II. Brit. J. Nutr., 14, 9 г-1 I3. 
Mitchel. II. H., Block R. J., 19+6. Some relationships between the aminoacid contents of proteins and their nutitrive values for the rat. J. Biol. Chem., 163, 599 .

NeHRING K., Bock II. D., 1902. Untersuchungen über die biolorische Wertigkeit von Eiweissfutterstoffen an Ratten. Arch. Tieremähr., 11, 370-392.

Nehrixi K., LAlbe W., Ig6r. Untersuchungen über die biologische Wertigkeit des liwweisses verschiedener Futterstoffe bein wachsenden Schwein. I. Z. Tierernähr, 16, 12 r-132.

NJAA L. R., I959. Biological value of herring meal protein. Urinary nitrogren excretion in relation to protein content of diet and food intake. Brit. J. Nutr., 13, I42-150.

Oser B. 1.,. 1959. In Albaxese A. A., Protein and amino-acid nulrition, 281-295. Acalenic Press, New York and London.

Ousterholt L. E., Snyder 1). ('r., ig62. In Hees li., Kreuzer R., Fish in mitrition, 303-300, Fishing news (books) Ltd, London.

Ravo N. T., ig6o. Biological evaluation of the factors affecting the protein quality of fish meals. Poultry Sci., 39, $+5-53$.

Kerat A., Pion R., Lotovon J.. IIEnry Y., Igf3. Étude du besoin azoté chez le porc en croissance. I. Utilisation de la farine de poisson à trois laux différsnts. Ann. Zootech. (sous presse).

SURE B., liASTERLING T., 1952. Evaluation of the biological value of the proteins in fish meals by the nitrogen retention method. J. Vur., 8, to1-405.

Tarr II. L. A., Bificy J., Marci B. E., 1954. The nutritive value of herring meals. I. The effect of heat. Poultry Sici, 33, 242-250.

Wilgus II. S. jr, Norris L. C., IIeuser (;. F., i935. IIaddock meal. Effect of manufacturing process upon nutritive value. Indust. Eng. Chem., 27, 419-422.

Zeiter S. Z., Charietetery (ieneviève, ig6i. Efficacité de quelques protides alimentaires chez le porc. I. Ann. Biol. anim. Bioch. Biophys., 1, 20-16. 